\title{
Fasudil, a Rho-kinase inhibitor, prevents intima-media thickening in a partially ligated carotid artery mouse model: Effects of fasudil in flow-induced vascular remodeling
}

\author{
XIANGYU ZHANG $^{1 *}$, TAO ZHANG $^{1 *}$, FU GAO $^{1}$, QINGLE LI $^{1}$, CHENYANG SHEN $^{1}$, \\ YANKUI LI ${ }^{2}$, WEI LI ${ }^{1}$ and XIAOMING ZHANG ${ }^{1}$ \\ ${ }^{1}$ Department of Vascular Surgery, Peking University People's Hospital, Beijing 100044; \\ ${ }^{2}$ Department of General Surgery, The Second Affiliated Hospital, \\ Tianjin Medical University, Tianjin 300000, P.R. China
}

Received August 28, 2014; Accepted May 19, 2015

DOI: $10.3892 / \mathrm{mmr} .2015 .4409$

\begin{abstract}
Vascular remodeling in response to hemodynamic alterations is a physiological process that requires coordinated signaling between endothelial, inflammatory and vascular smooth muscle cells (VSMCs). Extensive experimental and clinical studies have indicated the critical role of the Ras homolog gene family, member A/Rho-associated kinase (ROCK) signaling pathway in the pathogenesis of cardiovascular disease, where ROCK activation has been demonstrated to promote inflammation and remodeling through inducing the expression of proinflammatory cytokines and adhesion molecules in endothelial cells and VSMCs. However, the role of ROCK in flow-induced vascular remodeling has not been fully defined. The current study aimed to investigate the effect of the ROCK signaling pathway in flow-induced vascular remodeling by comparing the responses to partial carotid artery ligation in mice treated with fasudil (a ROCK inhibitor) and untreated mice. Intima-media thickness and neointima formation were evaluated by morphology. VSMC proliferation and inflammation of the vessel wall were assessed by immunohistochemistry. In addition, the expression levels of ROCK and the downstream effectors of ROCK, myosin light chain (MLC) and phosphorylated-MLC (p-MLC), were quantified by western blot analysis. Following a reduction in blood flow, ROCK1 and p-MLC expression increased in the untreated left common carotid arteries (LCA). Fasudil-treated
\end{abstract}

Correspondence to: Dr Xiaoming Zhang or Dr Wei Li, Department of Vascular Surgery, Peking University People's Hospital, 11 Xizhimen South Street, Beijing 100044, P.R. China

E-mail:rmyyxgwk@163.com

E-mail: mailtowei@qq.com

*Contributed equally

Key words: flow-induced vascular remodeling, Rho-associated kinase pathway, fasudil mice developed a significantly smaller intima-media thickness compared with the untreated mice. Quantitative immunohistochemistry of the fasudil-treated LCA indicated that there was a reduction in proliferation when compared with untreated vessels. There were fewer $\mathrm{CD} 45^{+}$cells observed in the fasudil-treated LCA compared with the untreated LCA. In conclusion, the expression of ROCK was enhanced in flow-induced carotid artery remodeling and ROCK inhibition as a result of fasudil treatment may attenuate flow-induced carotid artery remodeling.

\section{Introduction}

Vascular remodeling of the carotid arteries, characterized by carotid artery intima-media thickening (IMT), may be key in the pathogenesis of various cardiovascular diseases $(1,2)$. Recruitment and infiltration of inflammatory cells into the vascular walls, followed by the subsequent secretion of cytokines and growth factors leads to vascular smooth muscle cell (VSMC) proliferation and migration, which results in vascular remodeling (3). Abnormal shear stress, as well as various other stimuli, has been proposed as a critical factor that leads to vascular remodeling $(4,5)$. To further investigate this, a mouse model of IMT was developed, which was induced by partial ligation of the left common carotid artery (LCA) enabling regulation of the flow rates (6-8). Rho-associated kinase (ROCK) belongs to the AGC [protein kinase A (PKA)/protein kinase G/protein kinase C (PKC)] family of serine/threonine kinases, which is a key downstream effector of the small GTPase, Ras homolog gene family, member A (RhoA) (9). There are two ROCK isoforms, namely ROCK1 (also referred to as ROK $\alpha$ or p160ROCK) and ROCK2 (also referred to as ROKßor Rho-kinase). ROCK regulates actin cytoskeletal reorganization, smooth muscle contraction and cell migration $(10,11)$. Previous studies demonstrated that ROCK participates in numerous cardiovascular diseases, leading to abnormal contraction of smooth muscle, for example, cerebral and coronary vasospasm $(12,13)$, hypertension (14) and abdominal aortic aneurysm (9). Thus, ROCK is regarded as an important therapeutic target for the treatment 
of various cardiovascular conditions, including hypertension, atherosclerosis, heart failure and ischemic damage (10). However, whether the activity of ROCK is involved in flow-induced vascular remodeling remains unclear. In the current study, by investigating the effect of fasudil (the ROCK inhibitor) on vascular remodeling following a reduction in blood flow, the aim was to quantify the variations in ROCK expression in response to flow rate reduction, and investigate the specific role of fasudil in reducing recruitment of inflammatory cells, proliferation and migration of VSMCs.

\section{Materials and methods}

Animals. A total of 85 male mice (weight, 20-25 g; age, 8-weeks; Academy of Military Medical Sciences) were used in the current study and all procedures were approved by the Medical Ethics Committee of Peking University People's Hospital (Beijing; permit number, 2012-13). The control group consisted of 5 mice. The remaining 80 mice were randomly divided into 8 groups, with 10 mice per group. The were fed a standard diet and maintained in a specific-pathogen free environment in our hospital, with a $12 \mathrm{~h}$ light/dark cycle, and a house temperature of $20-28^{\circ} \mathrm{C}$.

Experimental protocol. Blood flow reduction in the LCA and flow measurements were performed as previously described (6). Briefly, each $\mathrm{C} 57 \mathrm{Bl} / 6 \mathrm{~J}$ mouse was anesthetized with ketamine and xylazine $(0.2 \mathrm{mg} / \mathrm{kg}$; Sigma-Aldrich, St. Louis, MO, USA) and were maintained at $37^{\circ} \mathrm{C}$ on a heating pad. Antibiotics (50 mg/kg cefazolin; Sigma-Aldrich) and $10 \mathrm{mg} / \mathrm{kg}$ analgesic (pentazocine via intramuscular injection; Sigma-Aldrich) were delivered subsequent to wound closure and sterilization with entoiodin (Sigma-Aldrich). The left external carotid artery distal to the thyroid artery and the left internal carotid/occipital artery were ligated with 8-0 sutures (Ethicon, Inc., Somerville, NJ, USA). A partial blood flow was maintained through the superior thyroid branch (15). In the sham group, vessel isolation was conducted with suture placed without ligation. The ROCK inhibitor, fasudil (Tianjin Chase Sun Pharmaceutical Co., Ltd., Tianjin, China) was dissolved in normal saline and injected intraperitoneally at $30 \mathrm{mg} / \mathrm{kg} / \mathrm{day}$ following LCA partial ligation until day 28. The mice were sacrificed through acute blood loss on days 3, 7, 14 and 28 for morphology and immunohistochemical analysis. The blood flow was measured in the carotid arteries using an ultrasonic flow meter (INC T101; Transonic Systems, Inc., Ithaca, NY, USA). The systolic blood pressure and heart rate of the conscious mice were monitored indirectly via the tail arteries (Visitech Systems, Apex, NC, USA).

Morphology. On days 3, 7, 14 and 28 following surgery, the vasculature was fixed by transcardial perfusion at $100 \mathrm{mmHg}$ with $25 \mathrm{ml} 10 \%$ paraformaldehyde (ZSGB-BIO, Beijing, China) in sodium phosphate buffer (pH 7.0; Sigma-Aldrich), as previously described (16). The left and right common carotid arteries were harvested and embedded in paraffin. Cross sections were stained with hematoxylin and eosin (H\&E) and elastic-van Gieson (EVG;ZSGB-BIO), and microscopic images were captured using a Olympus IX83 microscope (Olympus, Tokyo, Japan). These were analyzed using ImageJ software version 1.48u (National Institutes of Health, Bethesda, MD, USA). The circumference of the lumen, and the length of the internal elastic lamina (IEL) and the external elastic lamina (EEL) were determined by tracing along the luminal surface. The circumference of the lumen was used to represent the luminal area. Using ImageJ, the intimal area was determined as the area defined by the luminal surface and the IEL. The medial area was defined by the IEL and EEL. The adventitia area was defined by the EEL and vessel surface.

Immunohistochemical analysis. Immunohistochemical analysis of the paraffin-embedded vessel cross sections was performed as previously described (17). Sections (thickness, $3 \mu \mathrm{m}$ ) from the carotid arteries of the mice that were sacrificed on days 3, 7, 14 and 28 following ligation were obtained at $1 \mathrm{~mm}$ proximal to the ligature. The common carotid arteries were cut longitudinally and antibodies against cluster of differentiation (CD)45 monoclonal antibody (mAb; 1:50; cat. no. ZM-0213; ZSGB-BIO), CD68 mAb (1:100; cat. no. UM800047; OriGene Technologies, Inc., Rockville, MD, USA), proliferating cellular nuclear antigen (PCNA) mAb (1:50; cat. no. TA301137; Cell Signaling Technology, Inc., Danvers, MA, USA) and smooth muscle cell (SMC) $\alpha$-actin mAb (1:100; OriGene Technologies, Inc.) were used. Antigen retrieval was performed for cross sections with citric acid monohydrate (BD Pharmingen) at $95^{\circ} \mathrm{C}$ for $20 \mathrm{~min}$. Subsequently, the endogenous peroxidase activity was blocked by incubation for $20 \mathrm{~min}$ in methanol with3\% $\mathrm{H}_{2} \mathrm{O}_{2}$ (ZSGB-BIO), followed by incubation with the goat serum to mask any non-specific binding. For avidin-biotin-peroxidase complex (ABC) staining, the primary antibodies were incubated at $4{ }^{\circ} \mathrm{C}$ overnight, followed by secondary antibody (EnVision ${ }^{\mathrm{TM}}$; Dako, Glostrup, Denmark) incubation at room temperature for $60 \mathrm{~min}$ and $30 \mathrm{~min}$ with the ABC complex (Vector Elite; Vector Laboratories, Burlingame, CA, USA). The peroxidase-binding sites were visualized with 3,3'diaminobenzidine (Vector Laboratories), followed by hematoxylin counterstaining. En face preparations were evaluated with a Leica confocal microscope (TCS SP5 II; Leica, Microsystems GmbH, Wetzlar, Germany).

Western blotting. Vascular tissues were homogenized in ice-cold buffer $(50 \mathrm{mmol} / 1$ Tris, $\mathrm{pH} 7.4 ; 150 \mathrm{mmol} / \mathrm{l} \mathrm{NaCl}$; $0.5 \%$ Triton $\mathrm{X}-100 ; 1 \mathrm{mmol} / \mathrm{l}$ edetic acid; $1 \mathrm{~mol} / \mathrm{l}$ phenylmethylsulfonyl fluoride; and $5 \mathrm{mg} / \mathrm{l}$ aprotinin) and centrifuged at $14,000 \mathrm{x} \mathrm{g}$ at $4^{\circ} \mathrm{C}$ for $15 \mathrm{~min}$. The supernatants were then collected as total proteins, which were electrophoresed through 8\% SDS-PAGE (Sigma-Aldrich) and electrically transferred to a nitrocellulose membrane. The membrane was incubated at $4^{\circ} \mathrm{C}$ overnight in Tris(hydroxymethyl)aminomethane buffered saline (TBS) containing 5\% milk and the primary antibodies (1:1,000 dilution, Cell Signaling Technology, Inc.) against ROCK1, myosin light chain (MLC) and p-MLC. Subsequent to washing with TBS, the membranes were incubated with the secondary antibody (goat anti-rabbit IgG horseradish peroxidase conjugated) at room temperature for $1 \mathrm{~h}$. The protein expression levels of ROCK1 mAb (cat. no. 4035S), MLC mAb (cat. no. 3672S) and p-MLC mAb (cat. no. 3675S) were determined by western blotting where the relative quantity of protein was determined by densitometric analysis (Graigar DM3011; Graigar Technology Co., Ltd., Shenzhen, China). 
A

Sham

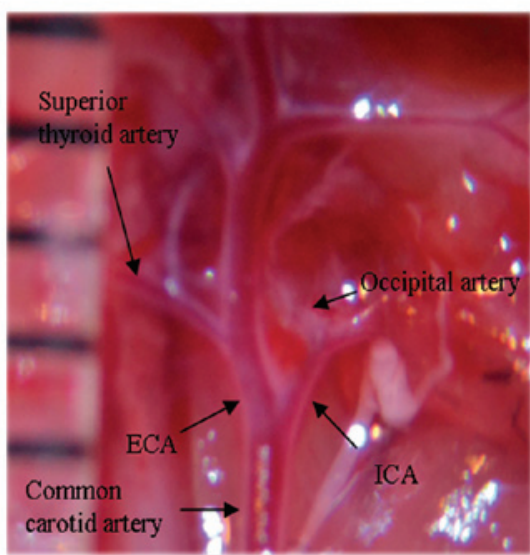

B

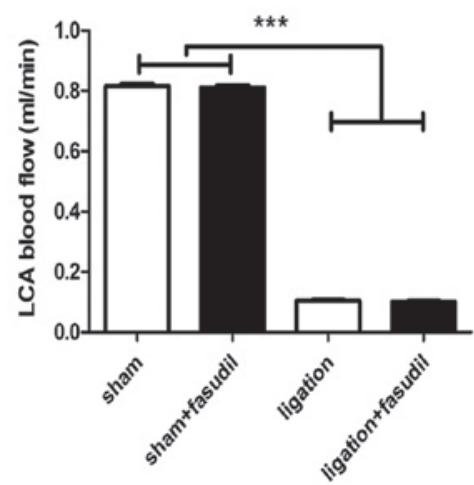

Ligation

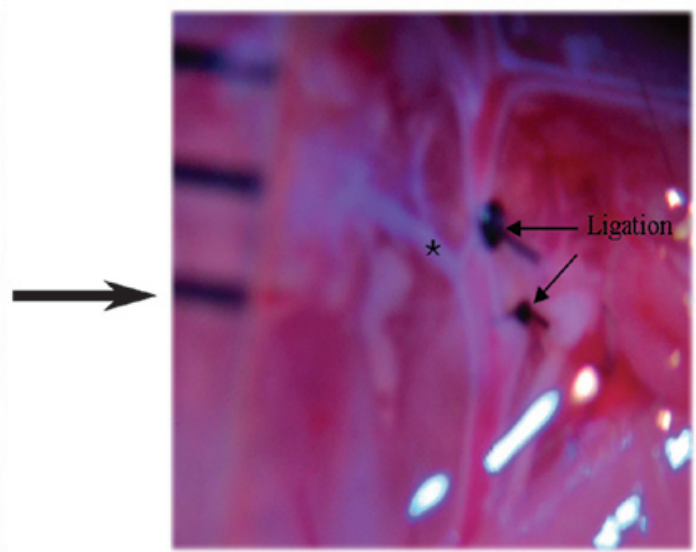

Figure 1. (A) Image of the ligation performed in the current study. Black arrows demonstrate the ligation points and the intact thyroid artery is highlighted by a black star. (B) The blood flow rate variations (reduction in LCA and increase in RCA) observed following partial ligation. Blood flow on the two sides was comparable in the sham and fasudil-treated groups prior and subsequent to ligation. Values are presented as the mean \pm standard error of the mean, $\mathrm{n}=8$ (number of vessels) ${ }^{* * *} \mathrm{P}<0.001$. LCA, left common carotid artery; RCA, right common carotid artery; ECA, external carotid artery; ICA, internal carotid artery.

The densitometric intensities of ROCK1, MLC and p-MLC were normalized to GAPDH values.

Statistical analysis. Data are represented as the mean \pm standard error of the mean. Comparisons for two groups were performed using Student's t-test. The differences among three or more groups were determined using one-way analysis of variance with Fisher's exact post-hoc test (Graphpad Software, Inc., La Jolla, CA, USA). P<0.05 was considered to indicate a statistically significant difference. Differences among groups were analyzed by repeated measures three times.

\section{Results}

Physiological and hemodynamic characteristics of C57B1/6J mice under partial ligation of carotid arteries. The mouse model used in the current study included ligation of the internal, external carotid and occipital arteries. Partial blood flow was maintained through the superior thyroid branch of the external carotid artery (Fig. 1A). This mouse model demonstrated significant alterations in blood flow patterns with a reduction of $\sim 80 \%$ in the LCA and a $40 \%$ increase in the right intact common carotid arteries (Fig. 1B). This result was consistent with previous studies (18-20). For fasudil-treated mice, almost

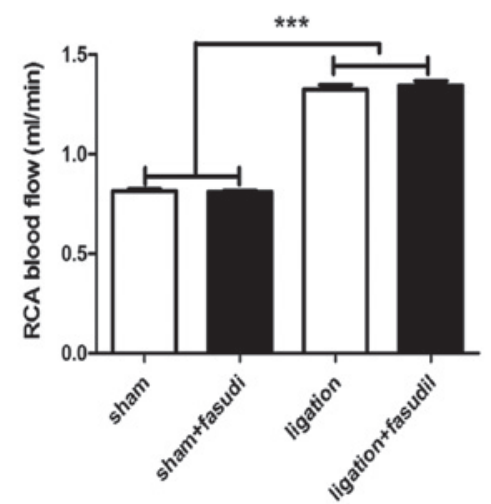

no influence on blood flow was observed prior or subsequent to LCA ligation (Fig. 1B) and abdominal administration of fasudil did not influence systemic blood pressure, which was consistent with previous reports (6). In summary, the LCA blood flow was significantly reduced in the mouse model, inducing intima-media thickness and neointima formation with almost no luminal alterations in LCAs.

ROCK signaling pathway activation in partially ligated vessels. To determine whether the ROCK signaling pathway participated in the flow-induced vascular remodeling, western blotting was used to analyze the protein expression levels of ROCK1, ROCK2 and their downstream effectors, MLC and p-MLC (Fig. 2). On days 7 and 28, significant increases in ROCK1 and p-MLC were observed in ligated LCA compared with in the sham groups $(\mathrm{P}<0.001)$ while no significant differences in ROCK2 and MLC were identified (Fig. 2A). In addition, fasudil was demonstrated to attenuate these variations, leading to significant reductions in protein expression of ROCK 1 and p-MLC in the fasudil-treated group compared with the untreated mice (Fig. 2B; $\mathrm{P}<0.05$ and $\mathrm{P}<0.01$, respectively).

Fasudil inhibits IMT and neointima formation in partially ligated LCA. In order to assess how blood flow affects 
A

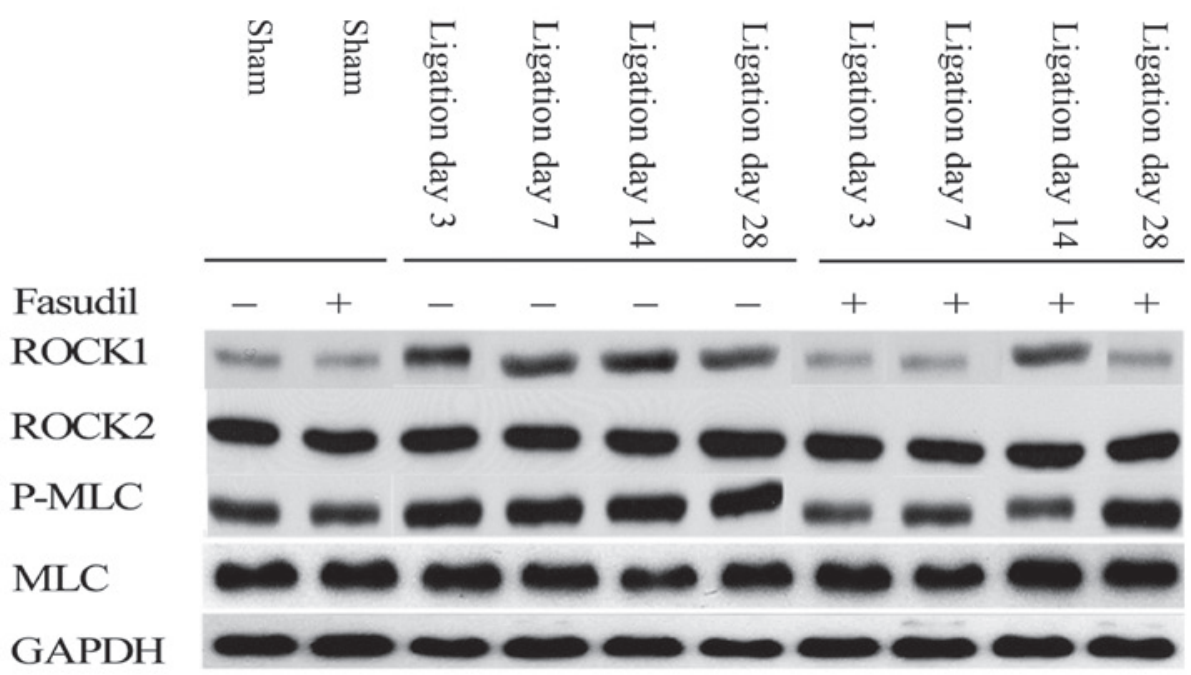

B
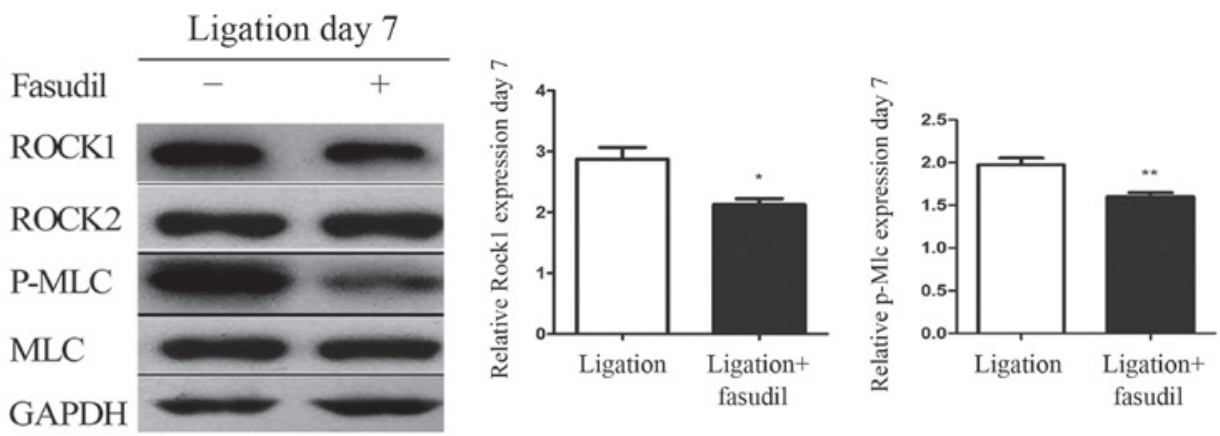

C

Ligation day 28
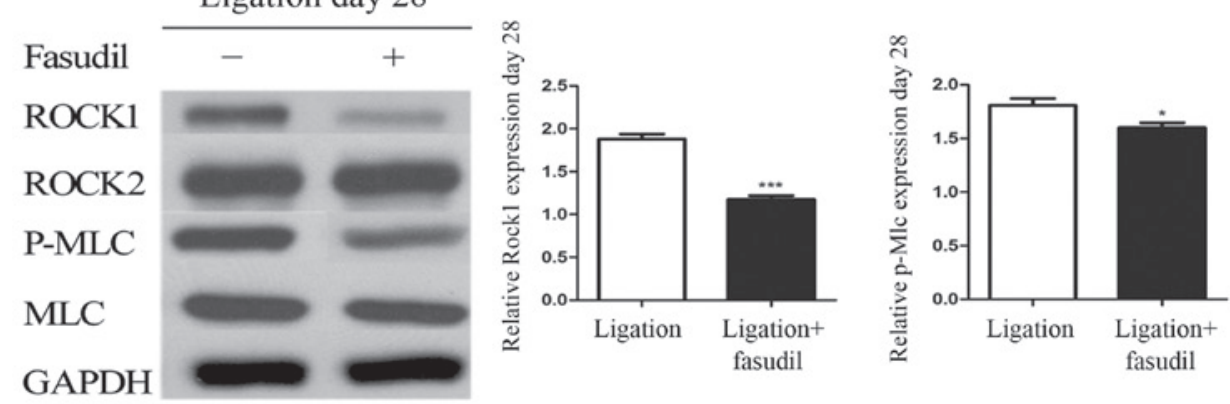

Figure 2. ROCK expression in sham and ligated mice. (A) Expression of ROCK isoforms and downstream markers of ROCK in LCA from fasudil-treated and untreated mice, with and without LCA partial ligation on days 3, 7, 14, and 28. Quantification of ROCK1, ROCK2, MLC and p-MLC in LCA from fasudil-treated and untreated mice on (B) day 7 and $(\mathrm{C})$ day 28 [ $\mathrm{n}=8$ (number of vessels); ${ }^{*} \mathrm{P}<0.05,{ }^{* *} \mathrm{P}<0.01$ and $\left.{ }^{* * * *} \mathrm{P}<0.001\right]$. ROCK, Rho-associated kinase; LCA, left common carotid artery; MLC, myosin light chain; p-MLC, phosphorylated-MLC.

arterial remodeling in vivo, the LCA partial ligation model was developed, enabling flow-induced remodeling in the common carotid arteries of fasudil-treated and untreated mice to be examined and compared. H\&E staining of the LCA vessel areas (Fig. 3A) demonstrated no differences in the sham fasudil-treated and untreated vessels. Furthermore, in response to a reduced flow in the LCA, the ligated LCA exhibited medial thickening and neointima formation. Seven days after ligation, no significant differences were observed between the fasudil-treated and untreated vessels. By contrast, 14 days subsequent to ligation, the areas of the vessel walls in the fasudil-treated mice were found to be markedly different from the sham mice. However, the untreated vessels exhibited clear medial thickening with little neointima formation (Fig. 3A). Twenty-eight days after ligation, the fasudil-treated mice exhibited a notable reduction in the media with a small quantity of neointima formation when compared with the untreated mice (Fig. 3A). Thus, ROCK inhibition may effectively reduce IMT and neointima formation that is induced by low blood flow in the carotid arteries. Through EVG staining, no significant alterations of the elastic lamina were observed, however, an increase in the media extracellular matrix was identified in the LCA (Fig. 3B). Extracellular matrix increase in the media was one of the most widespread and clear morphological features observed. On day 28 , the neointima developed in the two groups, more extensively in 


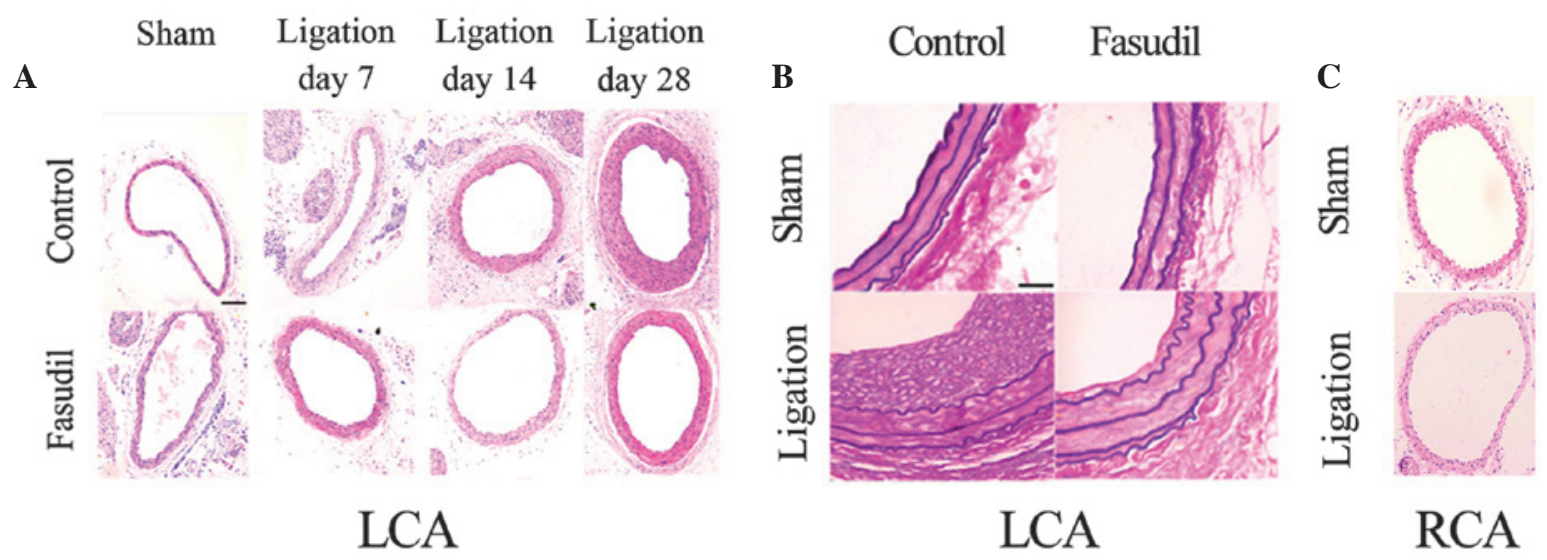

D
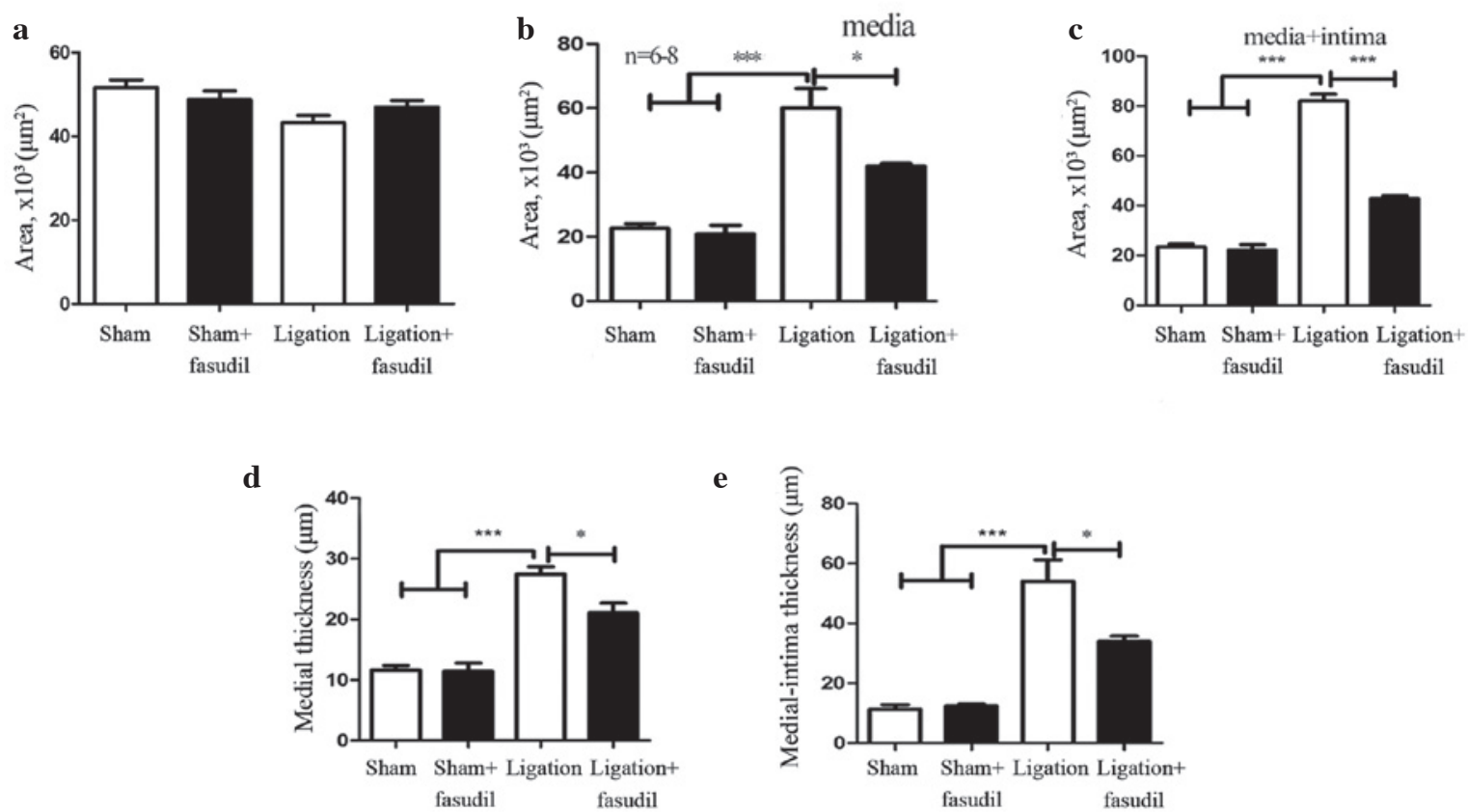

Figure 3. Morphology of the control and fasudil-treated carotid arteries following ligation. (A) Representative light microscopy images of hematoxylin and eosin-stained carotid artery cross sections obtained from the control and fasudil-treated mice. Medial thickening was observed in the control and fasudil-treated mice on day 7, and neointima formation was apparent on days 14 and 28 (magnification, x40; scale bar, $20 \mu \mathrm{m}$ ). (B) Elastic-van Gieson staining of carotid arteries from LCA cross-sections with or without fasudil treatment four weeks after ligation (sham, sham + fasudil, ligation and ligation + fasudil; magnification, $\mathrm{x} 40$; scale bar, $20 \mu \mathrm{m}$ ). (C) Representative light microscopy images of hematoxylin and eosin-stained RCA cross sections obtained from the sham and ligated mice (magnification, x40; scale bar, $20 \mu \mathrm{m}$ ). (D) Volumes of vessel components, including the lumen, media and media + intima, in addition to the vessel wall thickness of the media and media + intima. Black and white bars indicate fasudil-treated and untreated groups, respectively. Values are presented as the mean \pm standard error of the mean; $n=8$ (number of vessels). ${ }^{*} \mathrm{P}<0.05,{ }^{* * * *} \mathrm{P}<0.001$ (by analysis of variance). LCA, left common carotid artery; RCA, right common carotid artery.

the untreated mice than in the fasudil-treated mice (Fig. 3B). For fasudil-treated mice, the medial and intima + media areas were reduced by $\sim 28 \%(\mathrm{P}<0.05)$ and $45 \%(\mathrm{P}<0.001)$, respectively, compared with the untreated group (Fig. 3C). Medial and intima + media thickness was significantly reduced by $18 \%(\mathrm{P}<0.05)$ and $32 \%(\mathrm{P}<0.05)$, respectively, in the fasudil-treated group when compared with the untreated group (Fig. 3C). These data indicate that the ROCK inhibitor is capable of reducing the extent of hyperplasia following carotid ligation. No significant differences were observed in the lumenal area of the four groups (Fig. 3D).

Inhibition effect of fasudil on VSMC proliferation. Various studies have demonstrated that VSMC proliferation is a driving force for vascular remodeling in response to flow reduction $(6,21,22)$. To gain further insights into the mechanisms of ROCK signaling pathway-based vascular remodeling, the level of VSMC proliferation in the vascular wall was assessed by staining for PCNA. As presented in Fig. 4A, PCNA staining in the vascular wall was absent in the early stage following carotid artery ligation. On day 28, subsequent to ligation, in the untreated ligated mice the PCNA-positive cells had significantly increased and were observed to migrate into the neointima, when compared with the fasudil-treated ligated mice ( $\mathrm{P}<0.001$; Fig. 4A).

To characterize the phenotype of VSMCs, staining for SMC $\alpha$-actin expression was performed. On day 14 subsequent to ligation, $\alpha$-actin-positive cells were observed to 
A

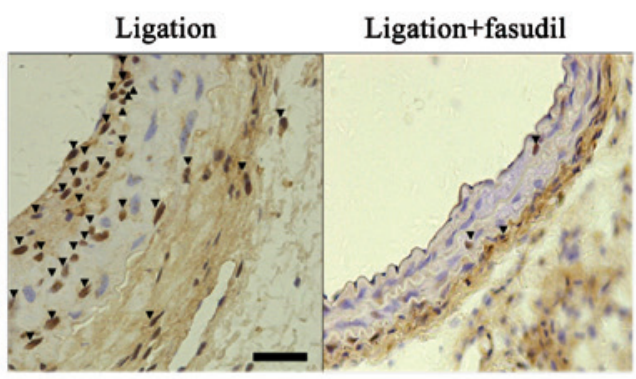

B

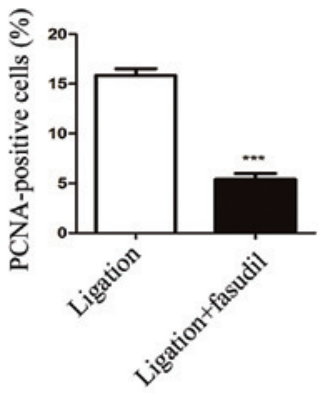

B Day 14

Day 28

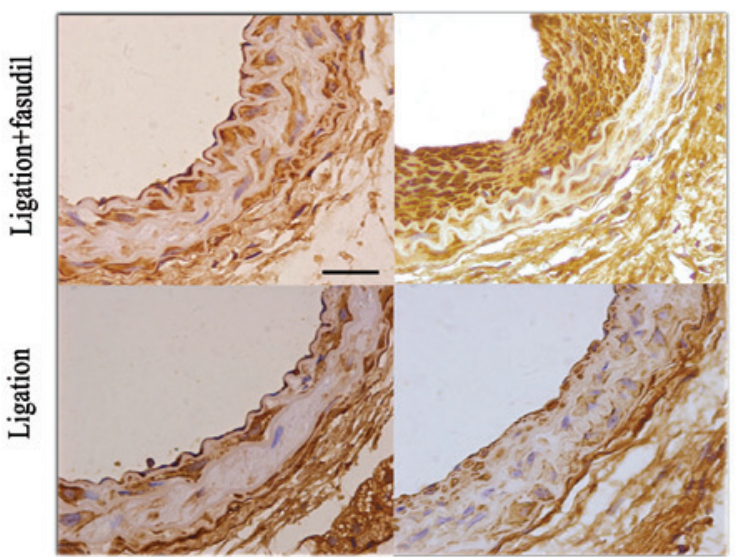

Figure 4. Flow-induced vascular remodeling is reduced in fasudil-treated mice. Proliferation and migration of vascular smooth muscle cells were identified by immunohistochemistry on paraffin-embedded sections of the carotid arteries from wild-type C57Bl/6J mice. (A) PCNA expression in the LCA of (a) untreated or (b) fasudil-treated arteries $\left(16.1 \pm 2.4 \%\right.$ vs. $\left.5.2 \pm 2.1 \% ;{ }^{* * * *} \mathrm{P}<0.001\right)$. Values are presented as the mean \pm standard error of the mean; $\mathrm{n}=8$ (number of vessels). (B) Smooth muscle cell $\alpha$-actin staining of LCA cross-sections from fasudil-treated and untreated mice 14 days and 28 days subsequent to ligation (vascular smooth muscle cells are stained brown; magnification, x40; scale bar, $20 \mu \mathrm{m})$. PCNA, proliferating cell nuclear antigen; LCA, left common carotid artery.

A

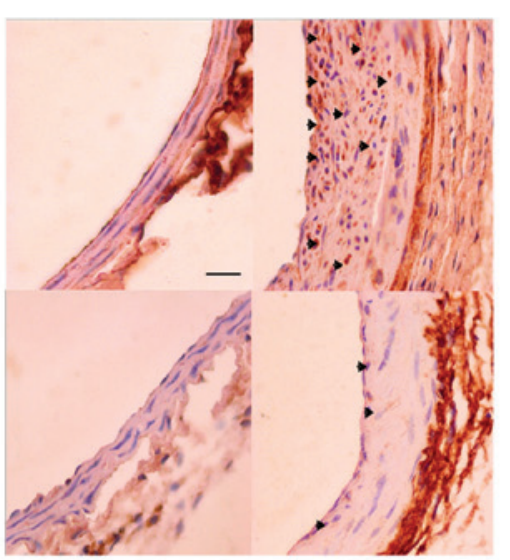

D

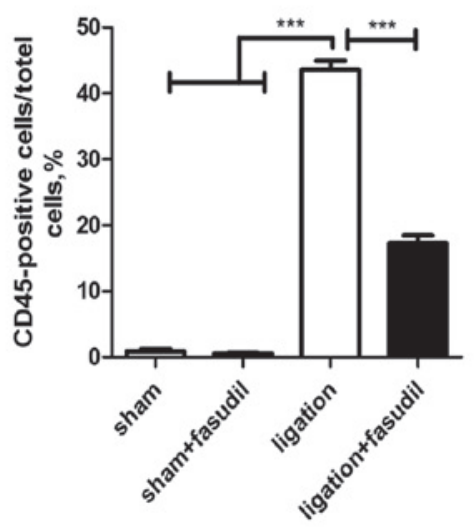

B

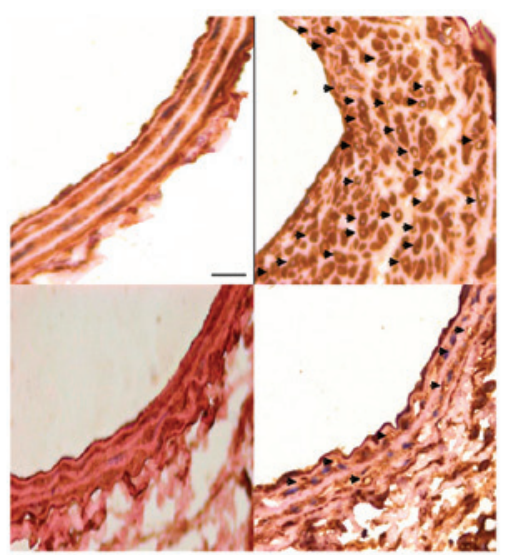

E

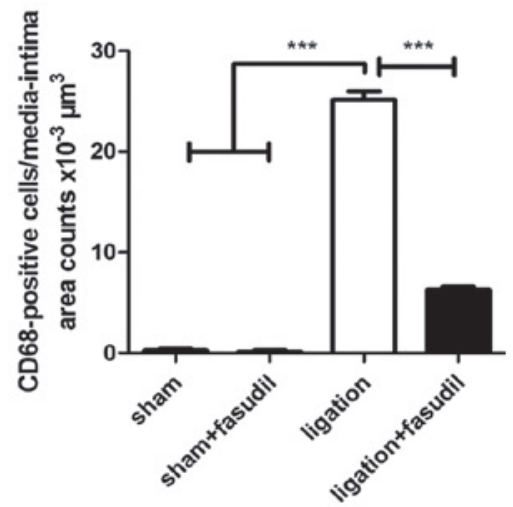

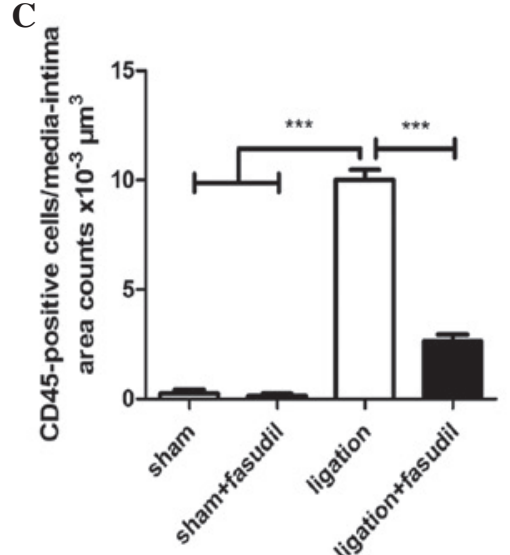

$\mathbf{F}$

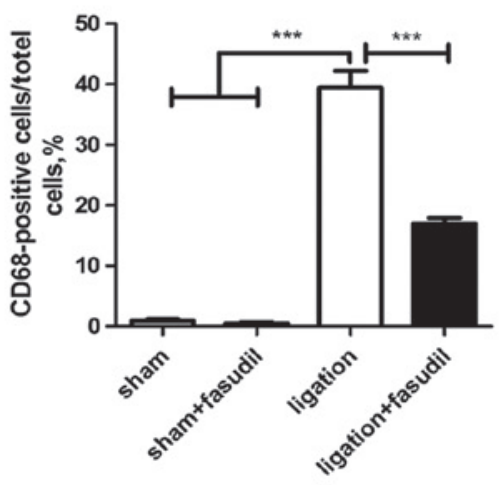

Figure 5. Flow-induced vascular remodeling is associated with the accumulation of inflammatory cells. The accumulation of leukocytes and macrophages was assessed by CD45 and CD68 immunohistochemistry (hematoxylin and eosin counterstaining), respectively. In the ligated mice without fasudil treatment, leukocyte accumulation was apparent in all the layers, while fewer leukocytes accumulated within the ligated mice that were treated with fasudil. (A) Positive cells are represented by black arrows (magnification, $\mathrm{x} 40$; scale bar, $20 \mu \mathrm{m}$ ). (B) Ligation of fasudil-treated mice demonstrated reduced macrophage accumulation in the vessel walls (magnification, 40x; scale bar, $20 \mu \mathrm{m}$ ). (C) Positive cells per area. (D) Positive cells per total cells. (E) Positive cells per area. (F) Positive cells per total cells. Values are presented as the mean \pm standard error of the mean, $\mathrm{n}=8$ (number of vessels). ${ }^{* * *} \mathrm{P}<0.001$. $\mathrm{CD}$, cluster of differentiation.

migrate to the intima-media area in the fasudil-treated and untreated vessels. On day 28 , subsequent to ligation, a markedly increased intensity of $\alpha$-actin-positive cells accumulated in the neointima area of the untreated ligated mice, whereas there was a reduced intensity of $\alpha$-actin staining in the media and neointima of the fasudil-treated mice (Fig. 4B). 
Fasudil treatment induced a reduction of inflammatory cell infiltration to the vascular wall, in the partially carotid ligated mouse model. Previous studies have demonstrated that the inflammatory response is important during vascular remodeling (6). Inflammatory cell interaction and recruitment to the vascular wall contributes to neointima formation and vascular inflammation (5). In the current study, inflammatory cells were identified by staining for the leukocyte antigen, CD45, which was followed by quantitative analysis using ImageJ software (23).

In the sham group, quantitative evaluation demonstrated that there were almost no $\mathrm{CD} 45^{+}$cells within the mouse intima-media area and few in the adventitia (Fig. 5A). Ligation notably increased the number of leukocytes in the vessel walls, where the majority of $\mathrm{CD} 45^{+}$cells were infiltrated to the neointima area and a small portion remained in the media area (Fig. 5A). For fasudil-treated mice, the number of CD45+ cells infiltrated into the vessel wall was markedly reduced (Fig. 5A).

Macrophages in the vessel wall were identified by staining for antigen CD68 (a macrophage marker). In the sham group, there were almost no $\mathrm{CD}^{+} 8^{+}$cells observed in the mouse intima-media area and adventitia (Fig. 5B). Ligation significantly increased the number of $\mathrm{CD}^{+} 8^{+}$cells present in the vessel walls, with a significant increase in the intima-media area, particularly in the neointima. For fasudil-treated mice, infiltration of $\mathrm{CD}^{+} 8^{+}$cells to the vessel wall was markedly reduced (Fig. 5B).

The graph in Fig. 5C demonstrates that the number of $\mathrm{CD} 45^{+}$cells infiltrated to the intima-media area in the ligated untreated vessel walls was $\sim 5$-fold that of the fasudil-treated vessels (Fig. 5C). The percentage of $\mathrm{CD} 45^{+}$cells among all the cells in the ligated vessel walls was $\sim 3$-fold that of the fasudil-treated vessels (Fig. 5D). In addition, the number of $\mathrm{CD} 8^{+}$cells infiltrated into the intima-media area in the ligated untreated vessel walls was $\sim 3$-fold that of fasudil-treated vessels (Fig. 5E). The percentage of $\mathrm{CD}^{+} 8^{+}$cells among all the cells in the ligated vessel walls was $\sim 2.5$-fold that of the fasudil-treated vessels (Fig. 5F). In summary, inhibition of the ROCK signaling pathway may reduce inflammatory cell infiltration to the vascular walls.

\section{Discussion}

Flow-induced vascular remodeling is a complex process, involving the expression of multiple genes and the interaction of multiple cell types (24). Previous studies based on inbred mouse or rat strains have demonstrated the role of genetic factors in vascular remodeling and IMT $(21,25)$. The observations of the current study provide the first evidence that ROCK is required for flow-induced remodeling and IMT. The important conclusions of the current study include: i) Protein expression levels of ROCK1 and p-MLC significantly increased in response to the blood flow reduction in LCA. ii) Fasudil prevents the process of flow-induced vascular remodeling by reducing IMT and neointima formation. iii) Fasudil inhibits proliferation of VSMCs in vascular remodeling. iv) Fasudil alleviates inflammatory cell infiltration into the vascular walls during the process of vascular remodeling.

The current study aimed to investigate the ROCK effects on flow-induced vascular remodeling based on partial ligation of the LCA in C57Bl/6J mice. The left external carotid, internal carotid and occipital arteries were ligated leaving the superior thyroid artery intact. Sham procedures consisted of suturing without ligation. This ligation reduces $\sim 80 \%$ of the flow rate in the LCA and increases the flow rate in the right common carotid arteries by $\sim 40 \%$ with the endothelium intact (18-20). The current study is, to the best of our knowledge, the first to investigate the effects of ROCK on flow-induced vascular remodeling. The positive effect of fasudil was previously demonstrated in animal models associated with vascular injury and arteriosclerosis in general (25-28). Previously, fasudil (136 or $213 \mathrm{mg} / \mathrm{kg} / \mathrm{day}$ ) was demonstrated to attenuate angiotensin II-induced abdominal aortic aneurysm in apolipoprotein $\mathrm{E}$ (apoE)-KO mice without affecting blood pressure (9). Furthermore, fasudil (30 mg/kg/day) was reported to prevent early atherosclerotic plaque formation and terminate lesion progression in apoE-KO mice (29). In the current study, fasudil (30 mg/kg/day) was injected intraperitoneally into mice, and almost no influence on blood pressure was noted (Fig. 1B). In the current study, abdominal administration of fasudil (the inhibitor of ROCK) was demonstrated to reduce IMT and neointima formation, inhibit cell proliferation and alleviate inflammation to the vessel walls. Thus, it was proposed that the inhibition of ROCK is able to attenuate flow-induced vascular remodeling.

Previous studies have demonstrated that the ROCK/MLC signaling pathway is extensively described in VSMCs (30-33) and ROCK1, rather than ROCK2, mediates leukocyte recruitment and neointima formation following vascular injury (34). In the present study, western blot analysis was used to quantify the relative quantity of proteins of partially ligated LCA with and without fasudil treatment. ROCK1 protein expression levels significantly increased in untreated ligated vessels when compared with vessels of the sham group. Subsequent to ligation, the expression levels of ROCK1 protein significantly reduced in fasudil-treated mice compared with untreated mice. A similar trend was observed for the expression of p-MLC. Thus it was concluded that ROCK is involved in the pathogenesis of vascular remodeling following flow reduction, and that ROCK inhibitors effectively attenuate flow-induced vascular remodeling.

In the current study, the mice subjected to 28 days of LCA partial ligation with or without fasudil treatment were observed to exhibit IMT and neointima formation in the vessel walls. Evaluated by H\&E and EVG staining, 28 days subsequent to ligation, fasudil-treated mice exhibited a significant reduction in media with a lower quantity of neointima formation when compared with untreated ligated mice (Fig. 3A and B). In addition, significantly reduced intima-media thickness and neointima formation in fasudil-treated mice were observed. These results indicated that inhibition of ROCK results in the attenuation of IMT and neointima formation following flow reduction, which is consistent with previous studies (34).

Migration and proliferation of VSMCs are important factors involved in flow-induced vascular remodeling. In the current study, the number of PCNA-positive cells in the medial and intimal areas was markedly reduced in the fasudil-treated mice when compared with their untreated counterparts (Fig. 4A). In addition, VSMCs in fasudil-treated vessels exhibited lower migration and proliferation capabilities compared with untreated vessels. These observations 
indicate that the inhibition of the ROCK signaling pathway reduces VSMC migration and proliferation. Furthermore, the reductions in VSMC proliferation in the neointima observed in fasudil-treated mice may result from the reduction in the inflammatory response of the vessel wall (for example, reduction in leukocyte recruitment, and the subsequent reduction in the release of inflammatory and growth stimuli) (Fig. 4A).

Vascular inflammation participates in flow-induced vascular remodeling, while ROCK1 has been proposed to serve a critical role in vascular inflammatory diseases (35). Upregulation of the active form of RhoA leads to a significant increase in inflammatory cell accumulation in the endothelial cell monolayer in the present study, which was prevented by administration of fasudil. Similarly, fasudil has been demonstrated to inhibit MCP-1-induced migration of leukocytes (36). In the current study, CD45 ${ }^{+}$cells were measured on days 14 and 28 subsequent to ligation, a greater number of $\mathrm{CD} 45^{+}$cells were observed to accumulate in the medial-intimal areas of the untreated mice, making up $50 \%$ of the total cell count. In the fasudil-treated mice, the $\mathrm{CD} 45^{+}$cell counts were significantly reduced to $\sim 15 \%$ of the total cell count $(\mathrm{P}<0.001$; Fig. 5D). Furthermore, $\mathrm{CD} 68^{+}$cells were almost absent in the sham vessels, were significantly increased in the partially ligated vessels, and this increase was partially reversed by fasudil treatment. Thus, ROCK signaling pathway inhibition prevents inflammatory cells from infiltrating the vessel walls in flow-induced vascular remodeling.

Concerning the limitations of the current study, as fasudil is a non-selective ROCK inhibitor, it may have additional non-selective effects (36-38), and at higher concentrations, fasudil may additionally inhibit serine/threonine kinases, such as PKA and PKC (39). In addition, no dose comparison was included in the present study.

In conclusion, the present study identified the involvement of the ROCK signaling pathway in flow-induced vascular remodeling, observing significant ROCK1 expression level increases in ligated LCA. p-MLC, as the activated downstream effector of ROCK, was also significantly increased in ligated mouse LCA. Fasudil, as a ROCK inhibitor, was demonstrated to reduce ROCK 1 and p-MLC expression levels in ligated mice and attenuate the process of flow-induced vascular remodeling (for example, IMT, VSMC migration, and proliferation and lymphocyte accumulation). The current study elucidated the role of ROCK signaling in the regulation of vascular remodeling, and highlighted the role of a ROCK inhibitor, fasudil, in the attenuation of the process of flow-induced vascular remodeling. Therefore, further characterization of ROCK signaling and its pharmacological modulation may lead to the development of novel therapeutic strategies for targeting flow-induced vascular remodeling.

\section{Acknowledgements}

This study was supported by the Peking University People's Hospital Research and Development Funds (grant no. 2118000537 to Dr. Wei Li), Specialized Research Fund for the Doctoral Program of Higher Education of China (grant no. 2109000075 to Dr Xiaoming Zhang) and the National Natural Science Foundation of China (grant no. 81470574 to Dr Wei Li).

\section{References}

1. Davis PH, Dawson JD, Riley WA and Lauer RM: Carotid intimal-medial thickness is related to cardiovascular risk factors measured from childhood through middle age: The Muscatine Study. Circulation 104: 2815-2819, 2001.

2. Cheng KS, Mikhailidis DP, Hamilton G and Seifalian AM: A review of the carotid and femoral intima-media thickness as an indicator of the presence of peripheral vascular disease and cardiovascular risk factors. Cardiovase Res 54: 528-538, 2002

3. Libby P: Inflammation in atherosclerosis. Nature 420: 868-874, 2002.

4. Pasterkamp G, Galis ZS and de Kleijn DP: Expansive arterial remodeling: Location, location, location. Arterioscler Thromb Vasc Biol 24: 650-657, 2004.

5. Bobik A and Tkachuk V: Metalloproteinases and plasminogen activators in vessel remodeling. Curr Hypertens Rep 5: 466-472, 2003.

6. Korshunov Va and Berk BC: Flow-induced vascular remodeling in the mouse: A model for carotid intima-media thickening. Arterioscler Thromb Vasc Biol 23: 2185-2191, 2003.

7. Post MJ, Borst $\mathrm{C}$ and Kuntz RE: The relative importance of arterial remodeling compared with intimal hyperplasia in lumen renarrowing after balloon angioplasty. A study in the normal rabbit and the hypercholesterolemic Yucatan micropig. Circulation 89: 2816-2821, 1994.

8. Wentzel JJ, Gijsen FJ, Stergiopulos N, Serruys PW, Slager CJ and Krams R: Shear stress, vascular remodeling and neointimal formation. J Biomech 36: 681-688, 2003.

9. Wang YX, Martin-McNulty B, da Cunha V, Vincelette J, Lu X, Feng Q, Halks-Miller M, Mahmoudi M, Schroeder M, Subramanyam B, et al: Fasudil, a Rho-kinase inhibitor, attenuates angiotensin II-induced abdominal aortic aneurysm in apolipoprotein E-deficient mice by inhibiting apoptosis and proteolysis. Circulation 111: 2219-2226, 2005.

10. Shimokawa $\mathrm{H}$ and Takeshita A: Rho-kinase is an important therapeutic target in cardiovascular medicine. Arterioscler Thromb Vasc Biol 25: 1767-1775, 2005.

11. Noma K, Oyama N and Liao JK: Physiological role of ROCKs in the cardiovascular system. Am J Physiol Cell Physiol 290: C661-C668, 2006.

12. Sato M, Tani E, Fujikawa $H$ and Kaibuchi K: Involvement of Rho-kinase-mediated phosphorylation of myosin light chain in enhancement of cerebral vasospasm. Circ Res 87: 195-200, 2000.

13. Masumoto A, Mohri M, Shimokawa H, Urakami L, Usui M and Takeshita A: Suppression of coronary artery spasm by the Rho-kinase inhibitor fasudil in patients with vasospastic angina. Circulation 105: 1545-1547, 2002.

14. Uehata M, Ishizaki T, Satoh H, Ono T, Kawahara T, Morishita T, Tamakawa H, Yamagami K, Inui J, Maekawa M, et al: Calcium sensitization of smooth muscle mediated by a Rho-associated protein kinase in hypertension. Nature 389: 990-994, 1997.

15. Chen $\mathrm{Z}$ and Tzima E: PECAM-1 is necessary for flow-induced vascular remodeling. Arterioscler Thromb Vasc Biol 29: 1067-1073, 2009.

16. Szendröi M, Labat-Robert J, Godeau G and Robert AM: Immunohistochemical detection of fibronectin using different fixatives in paraffin embedded sections. Pathol Biol (Paris) 31: 631-636, 1983.

17. Erami C, Zhang H, Tanoue A, Tsujimoto G, Thomas SA and Faber JE: Adrenergic catecholamine trophic activity contributes to flow-mediated arterial remodeling. Am J Physiol Heart Circ Physiol 289: H744-H753, 2005.

18. Chatzizisis YS and Giannoglou GD: Pulsatile flow: A critical modulator of the natural history of atherosclerosis. Med Hypotheses 67: 338-340, 2006.

19. Stone PH, Coskun AU, Yeghiazarians Y, Kinlay S, Popma JJ, Kuntz RE and Feldman CL: Prediction of sites of coronary atherosclerosis progression: In vivo profiling of endothelial shear stress, lumen and outer vessel wall characteristics to predict vascular behavior. Curr Opin Cardiol 18: 458-470, 2003.

20. Silver AE and Vita JA: Shear-stress-mediated arterial remodeling in atherosclerosis: too much of a good thing? Circulation 113: 2787-2789, 2006.

21. Korshunov VA and Berk BC: Strain-dependent vascular remodeling: The ‘Glagov phenomenon' is genetically determined. Circulation 110: 220-226, 2004.

22. Korshunov VA, Mohan AM, Georger MA and Berk BC: Axl, a receptor tyrosine kinase, mediates flow-induced vascular remodeling. Circ Res 98: 1446-1452, 2006. 
23. Phillips JW, Barringhaus KG, Sanders JM, Hesselbacher SE, Czarnik AC, Manka D, Vestweber D, Ley K and Sarembock IJ: Single injection of P-selectin or P-selectin glycoprotein ligand-1 monoclonal antibody blocks neointima formation after arterial injury in apolipoprotein E-deficient mice. Circulation 107 2244-2249, 2003

24. Korshunov VA, Schwartz SM and Berk BC: Vascular remodeling: Hemodynamic and biochemical mechanisms underlying Glagov's phenomenon. Arterioscler Thromb Vasc Biol 27: 1722-1728, 2007.

25. Ibrahim J, Miyashiro JK and Berk BC: Shear stress is differentially regulated among inbred rat strains. Circ Res 92: 1001-1009, 2003.

26. Matsumoto Y, Uwatoku T, Oi K, Abe K, Hattori T, Morishige K, Eto Y, Fukumoto Y, Nakamura K, Shibata Y, et al: Long-term inhibition of Rho-kinase suppresses neointimal formation after stent implantation in porcine coronary arteries: involvement of multiple mechanisms. Arterioscler Thromb Vasc Biol 24: 181-186, 2004

27. Miyata K, Shimokawa H, Kandabashi T, Higo T, Morishige K, Eto Y, Egashira K, Kaibuchi K, Takeshita A, et al: Rho-kinase is involved in macrophage-mediated formation of coronary vascular lesions in pigs in vivo. Arterioscler Thromb Vasc Biol 20: $2351-2358,2000$

28. Pearce JD, Li J, Edwards MS, English WP and Geary RL: Differential effects of Rho-kinase inhibition on artery wall mass and remodeling. J Vasc Surg 39: 223-228, 2004

29. Wu DJ, Xu JZ, Wu YJ, Jean-Charles L, Xiao B, Gao PJ and Zhu DL: Effects of fasudil on early atherosclerotic plaque formation and established lesion progression in apolipoprotein E-knockout mice. Atherosclerosis 207: 68-73, 2009.

30. Grimm M, Haas P, Willipinski-Stapelfeldt B, Zimmermann WH, Rau T, Pantel K, Weyand M and Eschenhagen T: Key role of myosin light chain (MLC) kinase-mediated MLC2a phosphorylation in the alpha 1-adrenergic positive inotropic effect in human atrium. Cardiovasc Res 65: 211-220, 2005.
31. Rajashree R, Blunt BC and Hofmann PA: Modulation of myosin phosphatase targeting subunit and protein phosphatase 1 in the heart. Am J Physiol Heart Circ Physiol 289: H1736-H1743, 2005.

32. Davis JS, Hassanzadeh S, Winitsky S, Lin H, Satorius C, Vemuri R, Aletras AH, Wen H and Epstein ND: The overall pattern of cardiac contraction depends on a spatial gradient of myosin regulatory light chain phosphorylation. Cell 107: 631-641, 2001.

33. Wettschureck $\mathrm{N}$ and Offermanns S: Rho/Rho-kinase mediated signaling in physiology and pathophysiology. J Mol Med (Berl) 80: 629-638, 2002.

34. Noma K, Rikitake Y, Oyama N, Yan G, Alcaide P, Liu PY, Wang H, Ahl D, Sawada N, Okamoto R, et al: ROCK1 mediates leukocyte recruitment and neointima formation following vascular injury. J Clin Invest 118: 1632-1644, 2008.

35. Ming XF, Viswambharan H, Barandier C, Ruffieux J, Kaibuchi K, Rusconi S and Yang Z: Rho GTPase/Rho kinase negatively regulates endothelial nitric oxide synthase phosphorylation through the inhibition of protein kinase B/Akt in human endothelial cells. Mol Cell Biol 22: 8467-8477, 2002.

36. Ohashi K, Nagata K, Maekawa M, Ishizaki T, Narumiya S and Mizuno K: Rho-associated kinase ROCK activates LIM-kinase 1 by phosphorylation at threonine 508 within the activation loop. J Biol Chem 275: 3577-3582, 2000.

37. Davies SP, Reddy H, Caivano M and Cohen P: Specificity and mechanism of action of some commonly used protein kinase inhibitors. Biochem J 351: 95-105, 2000.

38. Bain J, Plater L, Elliott M, Shpiro N, Hastie CJ, McLauchlan H, Klevernic I, Arthur JS, Alessi DR and Cohen P: The selectivity of protein kinase inhibitors: A further update. Biochem J 408: 297-315, 2007.

39. Liao JK, Seto M and Noma K: Rho kinase (ROCK) inhibitors. J Cardiovasc Pharmacol 50: 17-24, 2007. 